PROCEEDINGS OF THE

AMERICAN MATHEMATICAL SOCIETY

Volume 139, Number 12, December 2011, Pages 4461-4466

S 0002-9939(2011)11009-8

Article electronically published on April 5, 2011

\title{
FORMALITY OF PASCAL ARRANGEMENTS
}

\author{
MATTHEW MILLER AND MAX WAKEFIELD
}

(Communicated by Jim Haglund)

\begin{abstract}
In this paper we construct a family of subspace arrangements whose intersection lattices have the shape of Pascal's triangle. We prove that even though the intersection lattices are not geometric, the complex complement of the arrangements are rationally formal.
\end{abstract}

\section{INTRODUCTION}

Let $V$ be a complex vector space of dimension $\ell$. In this paper, a subspace arrangement $\mathcal{A}$ is a finite collection of linear subspaces of $V$, and we assume that there are no inclusions between the elements of $\mathcal{A}$. Let $L(\mathcal{A})$ be the intersection lattice of $\mathcal{A}$ defined by all possible intersections of elements from $\mathcal{A}$ ordered by reverse inclusion. We call $M(\mathcal{A}):=V \backslash \bigcup_{X \in \mathcal{A}} X$ the complement of $\mathcal{A}$.

In this paper the main characters are the following class of arrangements, which we call Pascal arrangements.

Definition 1.1. Let $n$ be a positive integer and let $\ell=2 n-1$. For $1 \leq k \leq n$ let $X_{k}$ be the subspace defined by

$$
X_{k}=\left\{\left(v_{1}, \ldots, v_{\ell}\right) \in \mathbb{C}^{\ell} \mid v_{k}=\cdots=v_{k+n-1}\right\} .
$$

Define the subspace arrangement $\mathcal{P}_{n}$ to be the collection $\left\{X_{1}, \ldots, X_{n}\right\}$.

The arrangements $\mathcal{P}_{n}$ are a certain type of hypergraph arrangement as defined by Björner in [1] and further studied by Björner and Welker in [2, Kozlov in 8], and Peeva, Reiner and Welker in [10. The authors came upon these arrangements through their recent work [9, in which we study a generalization of hypergraph arrangements. Also, the arrangements $\mathcal{P}_{n}$ were a main focus in a combinatorial study by Brockman and Sagan but were unfortunately not published (for reference see $[3]$ ).

An integral part of the study of the topology and combinatorics of subspace arrangements is that of the formality, or lack thereof, of the complex complement. The key work done by Yuzvinsky in 12 and by Feichtner and Yuzvinsky in [6] shows that if the intersection lattice of a subspace arrangement is geometric, then its complex complement is rationally formal. Both 12 and 6 rely on the wonderful models of De Concini and Procesi from 44. Later, Denham and Suciu in [5, Grbić

Received by the editors October 15, 2009.

2010 Mathematics Subject Classification. Primary 52C35; Secondary 55R80.

The second author has been supported by NSF grant No. 0600893, the NSF Japan program, and the Office of Naval Research.

(C)2011 American Mathematical Society Reverts to public domain 28 years from publication 
and Theriault in [7, and the authors in [9] all exhibited non-geometric subspace arrangements whose complex complements are not rationally formal.

The main result of this paper is that the complements of the $\mathcal{P}_{n}$ are, to the contrary, rationally formal in spite of their intersection lattices being far from geometric. In Section 2 we investigate the combinatorics of the arrangements $\mathcal{P}_{n}$. Then in Section 3 we prove that the complement is rationally formal.

\section{Combinatorics of $\mathcal{P}_{n}$}

2.1. The intersection lattice. Fix a positive integer $n$; then for each $k$ with $1 \leq k \leq n$ the subspace $X_{k}$ has dimension $n$. The $X_{k}$ correspond to the atoms of the intersection lattice which we denote by $k$. Now we compute intersections of the subspaces which correspond to the join of atoms of the lattice. Let $1 \leq k_{1}<\cdots<$ $k_{s} \leq n$. Then since the dimension of the vector space is $2 n-1$ and the dimension of $X_{k}$ is $n$, the intersection

$$
X_{k_{1}} \cap \cdots \cap X_{k_{s}}=X_{k_{1}} \cap X_{k_{s}}=\left\{\left(v_{1}, \ldots, v_{\ell}\right) \in \mathbb{C}^{\ell} \mid v_{k_{1}}=\cdots=v_{k_{s}+n-1}\right\}
$$

has dimension $n+k_{1}-k_{s}$ and the join is

$$
k_{1} \vee \cdots \vee k_{s}=k_{1} \vee k_{s} .
$$

We denote this subspace by $X_{\left(k_{1}, k_{s}\right)}$ and the corresponding element of the lattice by $\left(k_{1}, k_{s}\right)$. We rank the intersection lattice $L\left(\mathcal{P}_{n}\right)$ by codimension and note that this is not a ranking in the usual sense because the bottom element $V$ has rank 0 , but then the next level of the intersection lattice corresponding to all the atoms has rank $n-1$. Each level above this increases in rank by one from the previous level, and the maximal element $(1, n)$ has rank $2 n-2$. The $i^{\text {th }}$ level of $L\left(\mathcal{P}_{n}\right)$ for $n-1<i \leq 2 n-2$ consists of elements of the form $(a, b)$ where $b-a=i-n+1$. There are $2 n-i-1$ of these subspaces, and there are $n$ atoms.

Now we examine the structure of the lattice by considering which elements are less than a fixed element $(a, b)$. In the case when $b=a+1$ the element $(a, a+1)$ is greater than the atoms $a$ and $a+1$. For arbitrary $a$ and $b$ the element $(a, b)$ sits directly above $(a+1, b)$ and $(a, b-1)$. All other elements in the $(i-1)^{\text {st }}$ level will not be comparable to $(a, b)$, and all other elements less than $(a, b)$ will be less than either $(a+1, b)$ or $(a, b-1)$. Now the next proposition follows and justifies the name of these arrangements.

Proposition 2.1. The intersection lattice of $\mathcal{P}_{n}$ with the element $V$ removed has the shape of the top $n$ rows of Pascal's triangle.

Remark 2.2. Because of this shape, for $n \geq 3$ the intersection lattice $L\left(\mathcal{P}_{n}\right)$ is not geometric.

2.2. The characteristic polynomial. The characteristic polynomial has been a crucial invariant in the study of both hyperplane and subspace arrangements. In this subsection we compute the characteristic polynomials for $\mathcal{P}_{n}$. First we recall the definitions. For any subspace arrangement $\mathcal{A}$ the Möbius function $\mu: L(\mathcal{A}) \rightarrow \mathbb{Z}$ is defined recursively as $\mu(V)=1$ and

$$
\mu(X)=-\sum_{Y \subsetneq X} \mu(Y),
$$


where $X \in L(\mathcal{A})$. Then the characteristic polynomial of $\mathcal{A}$ is

$$
\chi(\mathcal{A}, t)=\sum_{X \in L(\mathcal{A})} \mu(X) t^{\operatorname{dim}(X)} .
$$

Turning our attention to $\mathcal{P}_{n}$ we see that $\mu\left(X_{i}\right)=-1, \mu\left(X_{(a, a+1)}\right)=1$, and $\mu\left(X_{(a, b)}\right)=0$ whenever $b-a>1$. Hence we have proved the following proposition.

Proposition 2.3. The characteristic polynomial of $\mathcal{P}_{n}$ is

$$
\chi\left(\mathcal{P}_{n}, t\right)=(n-1) t^{n-1}-n t^{n}+t^{2 n-1} .
$$

We illustrate the ideas for $n=4$ in the following example.

Example 2.4. Let $n=4$. Then $\ell=7$ and there are 4 subspaces $X_{1}, X_{2}, X_{3}$, and $X_{4}$. Figure 1 is a picture of the lattice with the corresponding lattice element at each vertex. The Möbius values of the atoms are all -1 , the Möbius values of the codimension 4 elements are 1 , and the Möbius values of the higher codimension elements are all 0 . Hence the characteristic polynomial is $\chi\left(\mathcal{P}_{4}, t\right)=3 t^{3}-4 t^{4}+t^{7}$.

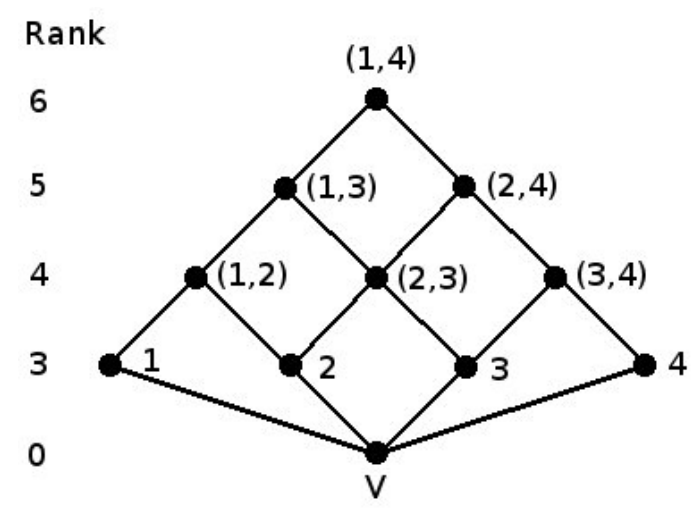

FiguRE 1. The intersection lattice of $\mathcal{P}_{4}$

\section{The complex complement}

In this section we compute the rational comology algebra of the complement of the arrangements $\mathcal{P}_{n}$ and show that the complements $M\left(\mathcal{P}_{n}\right)$ are rationally formal. The main tool is the relative atomic complex defined by Yuzvinsky in [11 and used by Feichtner and Yuzvinsky in [6] to prove that the complement of a subspace arrangement with a geometric intersection lattice is formal.

We now define the relative atomic complex. Let $\mathcal{A}=\left\{X_{1}, \ldots, X_{n}\right\}$ be a subspace arrangement and fix an order on these elements, $X_{1}<X_{2}<\cdots<X_{n}$. We associate the integer $s$ with the subspace $X_{s}$. Then we will use $\sigma=\left\{i_{1}, \ldots, i_{k}\right\} \subseteq\{1, \ldots, n\}$ to denote a subset of atoms in the intersection lattice of $\mathcal{A}$ such that $1 \leq i_{1}<i_{2}<$ $\cdots<i_{k}$. Let $D_{\mathcal{A}}$ be the differential graded algebra over $\mathbb{Q}$ with a generator $a_{\sigma}$ in degree $2 \operatorname{codim} \bigvee \sigma-|\sigma|$ for each $\sigma$. The differential is defined by

$$
d a_{\sigma}=\sum_{j: \bigvee \sigma \backslash i_{j}=\bigvee \sigma}(-1)^{j} a_{\sigma \backslash i_{j}},
$$


and the product structure is defined by

$$
a_{\sigma} a_{\tau}= \begin{cases}(-1)^{\varepsilon(\sigma, \tau)} a_{\sigma \cup \tau} & \text { codim } \bigvee \sigma+\operatorname{codim} \bigvee \tau=\operatorname{codim} \bigvee(\sigma \cup \tau), \\ 0 & \text { otherwise, }\end{cases}
$$

where $\varepsilon(\sigma, \tau)$ is the sign associated with the permutation that re-orders the linearly ordered $\sigma \cup \tau$ so that the elements of $\tau$ come after that of $\sigma$.

Feichtner and Yuzvinsky in [6] prove that the relative atomic complex, $D_{\mathcal{A}}$, is a rational model for the complex complement $M(\mathcal{A})$ by showing that it is quasiisomorphic to the rational models of $M(\mathcal{A})$ developed by De Concini and Procesi in 4 .

Returning to our examples we denote the relative atomic complex of $\mathcal{P}_{n}$ as $P_{n}$. Let $\sigma=\left\{k_{1}, \ldots, k_{s}\right\}$ and notice that the join $\bigvee \sigma$ is the lattice element $\left(k_{1}, k_{s}\right)$ and that the corresponding subspace has codimension $n-1+k_{s}-k_{1}$. Therefore, we have $\operatorname{deg}\left(a_{\sigma}\right)=2\left(n-1+k_{s}-k_{1}\right)-s$. Now let $\tau=\left\{j_{1}, \ldots, j_{r}\right\}$ with $k_{1} \leq j_{1}$. The join $\sigma \vee \tau$ is $\left(k_{1}, \max \left\{k_{s}, j_{r}\right\}\right)$, and that the corresponding subspace has codimension $\max \left\{k_{s}, j_{r}\right\}-k_{1}+n-1$.

Lemma 3.1. The only non-trivial product in $P_{n}$ is $a_{\{1\}} a_{\{n\}}=a_{\{1, n\}}$.

Proof. We first consider when $j_{r} \leq k_{s}$. In this case we have $\bigvee(\sigma \cup \tau)=\left(k_{1}, k_{s}\right)=$ $\bigvee \sigma$, which implies that $a_{\sigma} a_{\tau}=0$. Whence we may assume that $j_{r}>k_{s}$. Recall that $a_{\sigma} a_{\tau}$ is non-zero when

$$
n-1+k_{s}-k_{1}+n-1+j_{r}-j_{1}=\max \left\{k_{s}, j_{r}\right\}-k_{1}+n-1,
$$

and since $j_{r}>k_{s}$, this reduces to

$$
j_{1}-k_{s}=n-1 \text {. }
$$

This is true only when $j_{1}=n$ and $k_{s}=1$, which proves the lemma.

Define $T(k, j, s)$ to be the subcomplex of $P_{n}$ generated by all $a_{\sigma}$ where $\bigvee \sigma=$ $(k, j)$ and $|\sigma|=s$. If $\sigma$ satisfies these properties we say that $\sigma$ has type $(k, j, s)$.

Lemma 3.2. The algebra $\left(P_{n}, d\right)$ has a decomposition $\left(P_{n}, d\right)=\bigoplus_{(k, j)}(T(k, j, *), d)$ and $d(T(k, j, s)) \subset T(k, j, s-1)$.

Proof. Let $\sigma=\left\{k_{1}, \ldots, k_{s}\right\}$; the differential is determined by the formula

$$
d a_{\sigma}=\sum_{1<p<s}(-1)^{p} a_{\sigma \backslash k_{p}} .
$$

We restrict our attention to the subalgebras $(T(k, j, *), \hat{d})$, where $\hat{d}$ is the restriction of the differential of $P_{n}$ to $T(k, j, *)$ and more specifically to the sequence

$$
\cdots \rightarrow T(k, j, s+1) \rightarrow T(k, j, s) \rightarrow T(k, j, s-1) \rightarrow \cdots,
$$

where the maps are the restricted differential $\hat{d}_{s}: T(k, j, s) \rightarrow T(k, j, s-1)$.

Lemma 3.3. The differential $\hat{d}_{s}$ satisfies the inequality

$$
\operatorname{dim}\left(\operatorname{Im} \hat{d}_{s}\right) \geq\left(\begin{array}{c}
j-k-2 \\
s-3
\end{array}\right) .
$$


Proof. There is a one-to-one correspondence between the set of all $\sigma$ with type $(k, j, s)$ that begin with $k, k+1, \ldots$ and the set of $\sigma$ with type $(k, j, s-1)$ that do not contain $k+1$. These sets have order $\left(\begin{array}{c}j-k-2 \\ s-3\end{array}\right)$. The one-to-one correspondence is given by inserting (or deleting) the element $k+1$ from $\sigma$.

Lemma 3.4. The sequence (3.3) is exact for $s \geq 3$.

Proof. We show that the rank of the kernel of $\hat{d}_{s}$ is equal to the rank of the image of $\hat{d}_{s+1}$ :

$$
\begin{aligned}
\operatorname{dim}\left(\operatorname{Ker} \hat{d}_{s}\right) & =\operatorname{dim}(T(k, j, s))-\operatorname{dim}\left(\operatorname{Im} \hat{d}_{s}\right) \\
& \leq\left(\begin{array}{c}
j-k-1 \\
s-2
\end{array}\right)-\left(\begin{array}{c}
j-k-2 \\
s-3
\end{array}\right) \\
& =\left(\begin{array}{c}
j-k-2 \\
s-2
\end{array}\right) \\
& \leq \operatorname{dim}\left(\operatorname{Im} \hat{d}_{s+1}\right) .
\end{aligned}
$$

The second and fourth lines follow from Lemma 3.3 and the third line is Pascal's rule. Since $\hat{d}_{s}$ is a differential, the opposite inequality is also true.

Proposition 3.5. The rational cohomology algebra $H^{*}\left(\mathcal{P}_{n}\right)$ is isomorphic to the quotient of the polynomial algebra $\mathbb{Q}\left[\alpha_{\{j\}}, \alpha_{\{k, k+1\}}\right]$ by all products, where $1 \leq j \leq n$ and $1 \leq k \leq n-1$. The generators $\alpha_{\{k\}}$ have degree $2 n-3$ and $\alpha_{\{k, k+1\}}$ has degree $2 n-2$.

Proof. By Lemma 3.2 it suffices to prove the theorem for a fixed $T(k, j, *)$. Lemma 3.4 reduces the problem to the cases $s=1,2$. By inspection we have $d a_{\{k\}}=$ $d a_{\{k, j\}}=0$ and $d a_{\{k, i, j\}}=a_{\{k, j\}}$. Therefore, for $s=2$ the only elements that are not in the image of the differential are $a_{\{k, k+1\}}$.

Now we state and prove the main theorem.

Theorem 3.6. The complement of $\mathcal{P}_{n}$ is rationally formal.

Proof. We construct a quasi-isomorphism from $P_{n}$ to its homology. Define the map $F: P_{n} \rightarrow H\left(P_{n}\right)$ as the extension of

$$
F\left(a_{\sigma}\right)= \begin{cases}\alpha_{\sigma} & \text { if } \sigma=\{k\},\{k, k+1\}, \\ 0 & \text { if } \sigma \neq\{k\},\{k, k+1\}\end{cases}
$$

to an algebra map. We must check that $F$ does extend to an algebra map and that it is a quasi-isomorphism. By Lemma 3.1 the only product in $P_{n}$ is $a_{\{1\}} a_{\{n\}}=a_{\{1, n\}}$ and $F\left(a_{\{1\}}\right) F\left(a_{\{n\}}\right)=0=F\left(a_{\{1, n\}}\right)$ since there are no products in $H\left(P_{n}\right)$ by Proposition 3.5. Similarly, $F\left(d\left(a_{\sigma}\right)\right)=0=d\left(F\left(a_{\sigma}\right)\right)$ since $F$ is non-zero only on representatives of non-trivial homology classes. Lastly we note that $F\left(a_{\sigma}\right)=\alpha_{\sigma}=$ $\left[a_{\sigma}\right]$ when $\sigma=\{k\},\{k, k+1\}$, which shows that $F$ induces the identity map on homology. Thus $F$ is the desired quasi-isomorphism. 


\section{REFERENCES}

1. Anders Björner, Subspace arrangements, First European Congress of Mathematics, Vol. I (Paris, 1992), Progr. Math., vol. 119, Birkhäuser, Basel, 1994, pp. 321-370. MR.1341828 (96h:52012)

2. Anders Björner and Volkmar Welker, The homology of "k-equal" manifolds and related partition lattices, Adv. Math. 110 (1995), no. 2, 277-313. MR.1317619 (95m:52029)

3. William Brockman and Bruce E. Sagan, The $k$-consecutive arrangements, lecture slides, http://www.mth.msu.edu/ sagan/Slides/list.html.

4. C. De Concini and C. Procesi, Wonderful models of subspace arrangements, Selecta Math. (N.S.) 1 (1995), no. 3, 459-494. MR1366622(97k:14013)

5. Graham Denham and Alexander I. Suciu, Moment-angle complexes, monomial ideals and Massey products, Pure Appl. Math. Q. 3 (2007), no. 1, 25-60. MR.2330154

6. E. M. Feichtner and S. Yuzvinsky, Formality of the complements of subspace arrangements with geometric lattices, Zap. Nauchn. Sem. S.-Peterburg. Otdel. Mat. Inst. Steklov. (POMI) 326 (2005), Teor. Predst. Din. Sist. Komb. i Algoritm. Metody. 13, 235-247, 284. MR 2183223 (2007a:55021)

7. Jelena Grbić and Stephen Theriault, The homotopy type of the complement of a coordinate subspace arrangement, Topology 46 (2007), no. 4, 357-396. MR2321037

8. Dmitry N. Kozlov, A class of hypergraph arrangements with shellable intersection lattice, J. Combin. Theory Ser. A 86 (1999), no. 1, 169-176. MR1682970(2000e:52023)

9. Matthew S. Miller and Max Wakefield, Edge colored hypergraphic arrangements, Pure Appl. Math. Q. 8 (2012), no. 3, 761-783, to appear.

10. Irena Peeva, Vic Reiner, and Volkmar Welker, Cohomology of real diagonal subspace arrangements via resolutions, Compositio Math. 117 (1999), no. 1, 99-115. MR.1693007 (2001c:13021)

11. Sergey Yuzvinsky, Rational model of subspace complement on atomic complex, Publ. Inst. Math. (Beograd) (N.S.) 66(80) (1999), 157-164, Geometric combinatorics (Kotor, 1998). MR 1765044 (2002b:52026)

12. _ Small rational model of subspace complement, Trans. Amer. Math. Soc. 354 (2002), no. 5, 1921-1945 (electronic). MR.1881024(2003a:52030)

Department of Mathematics, Vassar College, Poughkeepsie, New York 12604

E-mail address: mamiller@vassar.edu

Department of Mathematics, United States Naval Academy, Annapolis, Maryland 21402

E-mail address: wakefiel@usna.edu 